to identify areas of commonality. Emerging themes included communication and information sharing, education and training, care planning, DNACPR, support and supervision for staff, and environment of care. From these themes, five key workstreams were developed (review of Trust guidance for care in last days of life, development of a staff EOLC intranet site, development of an EOLC planning toolkit, review compatibility of information platforms, education and training) and are currently being addressed by the EOLC working group.

Conclusion The comments collated from the consultation events provided a valuable source of rich qualitative data on the views of staff and have informed areas for development. The interest and engagement for this process confirmed the enthusiasm of staff, who valued the opportunity to shape future developments; many have since become involved in aspects of this work.

\section{ARE THERE INAPPROPRIATE PALLIATIVE PATIENT DEATHS IN EMERGENCY DEPARTMENTS (ED)? A RETROSPECTIVE STUDY LOOKING AT THE DEATHS IN A DISTRICT GENERAL HOSPITAL ED}

Hannah Fox, Matthew Doré, Derek Willis. Severn Hospice, Shrewsbury and Telford NHS Trust

\subsection{6/bmjspcare-2018-ASPabstracts.83}

Introduction Palliative patients sometimes present to emergency departments when dying and current policy aims to reduce such unnecessary admissions. This study provides a description of palliative care related deaths in an ED and an assessment of how many of these were preventable.

Methodology 32 consecutive deaths were reviewed in a district general ED department in Shropshire during 2016/2017. Death certification details, palliative status and DNACPR status were identified. We retrospectively judged whether their admission was potentially avoidable.

Results

Epidemiology

- Median age 83 years (range 40-95)

- 15 female patients (47\%).

Mode of death

- 12 patients died from respiratory pathology (37\%), 6 from cardiac (18\%), 6 from gastrointestinal (18\%), 3 from infection (9\%), 3 from stroke (9\%), 1 from cancer (3\%) and 2 were uncategorised.

- Frailty and palliative status

- Frailty and old age was documented on 10 death certificates (29\%), dementia on 3 (9\%).

- 4 patients (12\%) had oncological disease.

- 2 patients $(6 \%)$ were known to be palliative, 16 patients $(50 \%)$ were not. There is uncertainty regarding the palliative status of the remaining patients and clarification using the gold standard framework is ongoing.

- 25 patients were DNACPR (81\%).

We believe 3 admissions were preventable (9\%), 17 were unavoidable (53\%) and the remaining 12 are unclear (38\%).

Conclusion This study suggests that some palliative patients do present to ED and die, and that some of these admissions were avoidable. With ever increasing ED pressures we suggest it's imperative to understand the reasons, assess the scale, and find solutions to inappropriate palliative ED admissions.

\section{UNIFIED DO NOT ATTEMPT RESUSCITATION (UDNACPR) ORDERS - AN AUDIT OF COMMUNICATION BETWEEN DIFFERENT HEALTHCARE SETTINGS}

Imogen Sullivan, Tim Jackson. Salford Royal Hospital

10.1136/bmjspcare-2018-ASPabstracts.84

Background The use of unified Do Not Attempt Resuscitation ( 'uDNACPR') orders aims to facilitate effective communication of DNA-CPR decisions between primary and secondary care. The document remains valid in primary and secondary care facilities and ensures continuity of care. The Salford uDNACPR policy reinforces the importance of communication of such decisions with all healthcare professionals involved in the patients care.

Aims/objectives To review the quality of communication of uDNACPR decisions with General Practitioners (GPs), out of hours services and the North West Ambulance Service (NWAS) on discharge from hospital.

Method A retrospective audit included 26 patients who had a uDNACPR order introduced whilst an inpatient at Salford Royal Hospital. Data was collected from the electronic patient record, uDNACPR paper forms, GP practices, out of Hours services and the NWAS Electronic Referral Information Sharing System: 'ERISS'.

Results In 33\% of cases, GPs had no record of uDNACPR decisions. The NWAS had no record of uDNACPR orders in $81 \%$ of cases, and Out of Hours services had records of only $1 / 26$ orders.

Conclusion Current communication of uDNA-CPR decisions to community providers is inconsistent and there is clearly room for improvement. This may result in patients receiving futile and undesired interventions. One key factor identified for poor communication of uDNACPR orders was poor knowledge amongst healthcare professionals on communication channels for such decisions. Work is currently being done to facilitate and support communication to different providers on discharge from secondary care. This includes education sessions on electronic systems such as EPaCCS, discussions with the individuals responsible for 'electronic note templates' within the hospital electronic patient record and involvement in work aiming to introduce a 'Medical intraoperatively gateway' (MIG) allowing GP/hospital/other systems to communicate and share information.

\section{ANTICIPATORY PRESCRIBING PRACTICE AT A DISTRICT GENERAL HOSPITAL: A SERVICE EVALUATION}

Ayla Newton, Denise Dunsire, Brigid Purcell, Maria King, Penny McNamara, Richella Ryan. Bedford Hospital, Sue Ryder Hospice - Moggerhanger

\subsection{6/bmjspcare-2018-ASPabstracts.85}

Background NICE guidelines (NG31, 2015) advise that suitable anticipatory medicines (AMs) are prescribed as early as possible for people likely to need symptom control in the last days of life. To date, there has been limited evaluation of this practice. This study aimed to characterise anticipatory prescribing (AP) at a district general hospital in relation to three main 